\title{
Study of the effect of glycemic control on the diabetes- related distress in a sample of Egyptian patients with diabetes mellitus
}

\author{
Hamed $\mathrm{MS}^{1 *}$, Ibrahim NA ${ }^{1}$, Ali HM${ }^{1}$ and Kheir $\mathrm{CG}^{2}$ \\ ${ }^{1}$ Internal Medicine \& Endocrinology Department, Faculty of Medicine, Ain Shams University, Cairo, Egypt \\ ${ }^{2}$ Endocrinology Department, Ain Shams University, Cairo, Egypt
}

\begin{abstract}
Background: Living successfully with diabetes mellitus requires the very large task of managing a serious chronic condition. The tasks associated with diabetes can be complex and demanding and most people in their life journey with diabetes report great frustration with the burden of the disease.

Aim: Study of the effect of Glycemic Control on the Diabetes-Related Distress in a sample of Egyptian patients with Diabetes mellitus.

Patients and methods: this a cross sectional study conducted on 350 patients with T2DM attending the outpatient clinic of endocrinology of El Agouza Police Hospital.

Fasting blood glucose, Post prandial blood glucose, HbA1c, hs-CRP, Creatinine, BUN, SGPT and SGPT were assessed, BMI, Waist /Hip Ratio, also Diabetes Distress Screening Scale Questionnaire was completed by the patients.

Results: There is a statistically significant positive relationship between HbA1c and Total DDS score, Emotional burden, Physician related distress, Regimen related distress, and Interpersonal distress with P-value 0.000, 0.000, 0.000, 0.032, and 0.001 respectively.
\end{abstract}

Conclusion: Diabetes related Distress is associated with poor glycemic control and also inflammation.

Abbreviations: BMI: body mass index; CRP: C reactive protein; DDSS: Diabetes Distress Screening Scales; DKA: diabetic ketoacidosis; HbA1c: hemoglobin A1c; FBS: fasting blood sugar; T2DM: type 2 diabetes mellitus; 2hpp: 2hour post prandial; PVD: peripheral vascular disease.

\section{Introduction}

Diabetes mellitus is a group of metabolic diseases characterized by hyperglycemia resulting from defects in insulin secretion, insulin action or both. The chronic hyperglycemia of diabetes is associated with long term damage, dysfunction, and failure of various organs especially the eyes, kidneys, nerves, heart, and blood vessels [1].

Emotional distress is common in persons with diabetes, It is associated with poorer glycemic control, reduced quality of life, and higher mortality rates [2]. Emotional distress can either be generic, defined as depressive and/or anxiety symptoms not related to a specific cause, or diabetes-specific, that is, directly related to the experience of living with diabetes mellitus, such as fear of complications or worrying about the disease and its direct consequences for daily life [3].

Diabetes Distress is not a complication of diabetes, the emotional side of diabetes is simply part of having diabetes. An individual may not be feeling particularly distressed at one point in time, but become upset and distressed at another point in time, perhaps around the time of the emergence of a complication, a switch in medication, or a change in a healthcare provider - there are all kinds of things that can be distressing about diabetes [4].

Diabetes distress can be evaluated using the Diabetes Distress Screening Scales (DDSS), "The DDSS is a validated survey instrument that assesses diabetes-related emotional distress. Today, we have multiple distress scales for a variety of different people connected to diabetes.

Diabetes Distress is common and worldwide may affect as many as $40 \%$ of people diagnosed with diabetes. Diabetes distress, among other affective disorders has been shown to increase over time in individuals with type 2 diabetes [5] (Figure 1).

\section{Aim of the work}

Study of the effect of Glycemic Control on the Diabetes-Related Distress in a sample of Egyptian patients with Diabetes mellitus

${ }^{\star}$ Correspondence to: Mohamed Saad Hamed, Professor of Internal Medicine \& Endocrinology Department, Faculty of Medicine, Ain Shams University, Cairo, Egypt, E-mail: hanan.mahmoud29@yahoo.com

Key words: Diabetes Distress, Glycemic Control

Received: August 13, 2019; Accepted: September 12, 2019; Published: September 16, 2019 


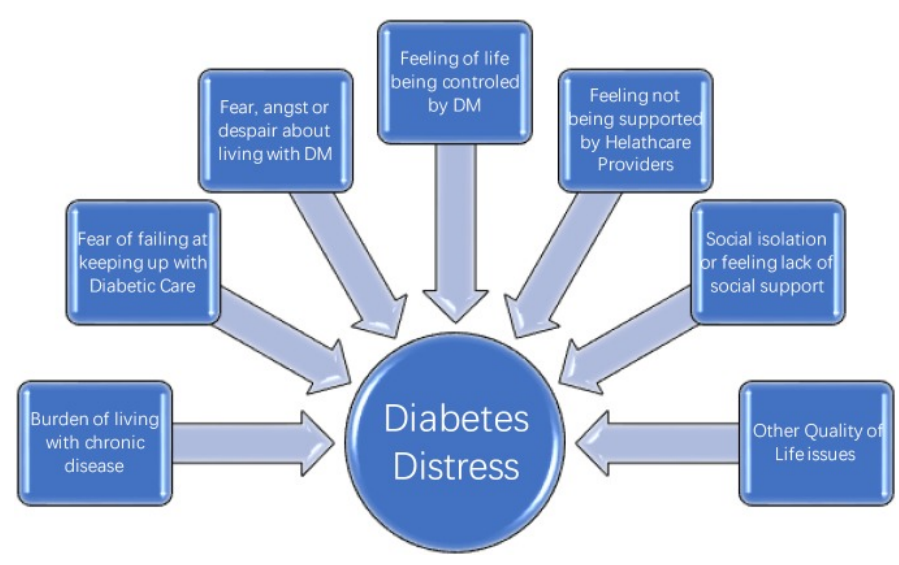

Figure 1. Understanding diabetes distress; a schematic representation

\section{Patients and methods}

This study was conducted on 350 patients with T2DM, their age ranged from 33 to 65 years, with duration of diabetes more than 5 year with exclusion of any other chronic disease as autoimmune, endocrinal diseases or chronic kidney, liver diseases, selected from the outpatient clinic of endocrinology of El Agouza Police Hospital, during the period from between June 2017 to June 2018 after signing an informed consent for all participant.

All participants subjected to full medical history emphasizing on the duration of diabetes mellitus, treatment, diabetic complications (regular fundus examination, puffiness of eye lids, frothy urine, diabetic foot, any vascular interventions, tingling, and cerebrovascular accidents, claudication pain), co-morbid conditions, anti-dyslipidemia drugs and smoking. All patients subjected to full clinical examination including: Anthropometric measurements of weight, height, BMI, waist circumference and waist/hip ratio, measurement of blood pressure, carotid and femoral bruit, signs of heart failure, foot ulcers, atrophic changes and neurological examination.

\section{Laboratory and diabetes distress screening scale}

Fasting plasma glucose, two hours post prandial plasma glucose, HbAlc, Serum creatinine, AST, ALT, hs-CRP and Albumin/creatinine ratio.

Diabetes Distress Screening Scale is a 17 -item scale that captures four critical dimensions of distress: Emotional burden, Regimen distress, Interpersonal distress and Physician distress. It was first published in 2005, it has been used widely around the world as a clinical instrument for opening conversation with one's patients as well as a critical outcome measures in numerous studies. In this study we used an Arabic version of the Diabetes Distress Screening Scale. It yields a total diabetes distress scale score plus 4 sub scale scores, each addressing a different kind of distress. To score, simply sum the patient's responses to the appropriate items and divide by the number of items in that scale. Scores range from 1 to 6 , we consider a mean item score of 3 or higher (moderate distress) as a level of distress worthy of clinical attention.

\section{Results}

The present study revealed that there is a statistically nonsignificant correlation between gender and Diabetes Distress Scale score. As shown in Table 1 and statistically significant negative correlation between age of the patients (P-value 0.002) and Total DDS score as shown in Table 2 also there is statistically non-significant correlation between BMI and Diabetes Distress Scale score. As shown in Table 2.

The current study showed that there is a highly statistically significant positive correlation between Total DDS score and glycemic profile (FBS, 2Hpp, HbAlc) (P-value 0.000) also with an inflammatory marker (CRP) with (P-value 0.000), Albumin/Creatinine ratio with (P-value 0.008) as shown in Table 3.

The current study showed that there is a statistically highly significant correlation between the Total DDS score and the presence of diabetic complications either acute or chronic diabetic complications; DKA, hypoglycemia with (P- value 0.000$)$, Diabetic retinopathy with (P-value 0.019), Diabetic Neuropathy (P-value 0.000), cerebrovascular complications with (P-value 0.000), peripheral vascular disease with (P-value 0.001) while there is non-significant relationship between the Total DDS score and the presence of diabetic Cardiovascular complications. As shown in Table 4.

In the present study we found that the included patients were have the percentage $84.3 \%$ showed Mild distress as regard the Total DDS score while $15.7 \%$ of the patients showed Moderate distress as seen in Figure 2.

On comparing between the mild and moderate distress groups as regard the parameter of glycemic control (HbAlc), inflammatory marker (CRP) and the presence of diabetic complications either acute or chronic we found that the moderate distress group have higher HbAlc $(9.11+2.22)$, CRP $(3.66+1.25)$ than the mild distress group as HbAlc $(8.01+2.2)$, CRP $(3.24+1.04)$ with a highly statistical significant difference (P-value $0.000,0.008)$ respectively as shown in Table 5.

And by the logistic regression analysis we found that there was highly statistically significant association found between moderate distress by total DDS score and retinopathy, neuropathy, nephropathy with Odds ratio (OR) and 95\% confidence interval (CI) of 2.836 (1.562 - 5.150), 2.633 (1.382 - 5.020) and 1.008 (1.003 - 1.013),) respectively. Also the table shows that there was statistically significant association found between moderate distress by total DDS score and DKA, hypoglycemia, cardiovascular, cerebrovascular and PVD with OR $(95 \%$ CI) of 2.355 (1.096 - 5.062), 2.758 (1.225 - 6.207), 2.107 (1.141 - 3.891),

Table 1. Correlation between age and BMI of the patients and total DDS score

\begin{tabular}{|c|c|c|}
\hline \multirow{2}{*}{ Age } & \multicolumn{2}{|c|}{ Total DDS score } \\
\cline { 2 - 3 } & $\mathbf{r}$ & $\boldsymbol{P}$-value \\
\hline BMI & $-0.168^{* *}$ & 0.002 \\
\hline
\end{tabular}

Table 2. Correlation between total DDS score and glycemic profile

\begin{tabular}{|c|c|c|c|c|c|c|}
\hline & Female & Male & \multirow{2}{*}{$\begin{array}{c}\text { Test } \\
\text { value• }\end{array}$} & \multirow{2}{*}{$P$-value } & \multirow{2}{*}{ Sig. } \\
\hline & & No. $=$ & No. $=$ & & & \\
\hline \multirow{3}{*}{$\begin{array}{l}\text { Total } \\
\text { DDS } \\
\text { score }\end{array}$} & Mean \pm SD & $2.28 \pm 1.08$ & $2.20 \pm 0.95$ & \multirow{3}{*}{0.711} & \multirow{3}{*}{0.478} & \multirow{3}{*}{ NS } \\
\hline & Range & $1-5.82$ & $1-6$ & & & \\
\hline & Range & $1-6$ & $1-6$ & & & \\
\hline
\end{tabular}

Table 3. Correlation between total DDS score and glycemic profile (FBS, $2 \mathrm{Hpp}, \mathrm{HbA1c}$ ) an inflammatory marker (CRP), albumin/creatinine ratio

\begin{tabular}{|l|c|c|}
\hline \multirow{2}{*}{ FBS } & \multicolumn{2}{|c|}{ Total DDS score } \\
\cline { 2 - 3 } & $\mathrm{r}$ & $\mathrm{p}$-value \\
\hline 2Hpp & $0.209^{* *}$ & 0.000 \\
\hline HbA1c & $0.228^{* *}$ & 0.000 \\
\hline CRP & $0.209^{* *}$ & 0.000 \\
\hline Alb/Creat & $0.350^{* *}$ & 0.000 \\
\hline
\end{tabular}


Table 4. Correlation between the total DDS score and the presence of diabetic complications either acute or chronic diabetic complications

\begin{tabular}{|c|c|c|c|c|c|c|}
\hline & & \multicolumn{2}{|c|}{ Total DDS score } & \multirow{2}{*}{ Test value• } & \multirow{2}{*}{ P-value } & \multirow{2}{*}{ Sig. } \\
\hline & & No & YES & & & \\
\hline \multirow{2}{*}{ DKA } & Mean \pm SD & $2.16 \pm 1.02$ & $2.82 \pm 0.65$ & \multirow{2}{*}{-4.010} & \multirow{2}{*}{0.000} & \multirow{2}{*}{ HS } \\
\hline & Range & $1-6$ & $1.59-4.06$ & & & \\
\hline \multirow{2}{*}{ Hypoglycemia } & Mean \pm SD & $2.17 \pm 1.02$ & $2.89 \pm 0.52$ & \multirow{2}{*}{-3.932} & \multirow{2}{*}{0.000} & \multirow{2}{*}{ HS } \\
\hline & Range & $1-6$ & $2.29-4.06$ & & & \\
\hline \multirow{2}{*}{ Cardiovascular complications } & Mean \pm SD & $2.17 \pm 1.13$ & & \multirow{2}{*}{-1.602} & \multirow{2}{*}{0.110} & \multirow{2}{*}{ NS } \\
\hline & Range & $1-6$ & $1.35-4.06$ & & & \\
\hline \multirow{2}{*}{ Diabetic retinopathy } & Mean \pm SD & $2.16 \pm 1.09$ & $2.44 \pm 0.69$ & \multirow{2}{*}{-2.357} & \multirow{2}{*}{0.019} & \multirow{2}{*}{$\mathrm{S}$} \\
\hline & Range & $1-6$ & $1.35-4.06$ & & & \\
\hline \multirow{2}{*}{ Cerebrovascular complications } & Mean \pm SD & $2.17 \pm 1.02$ & $2.94 \pm 0.54$ & \multirow{2}{*}{-3.942} & \multirow{2}{*}{0.000} & \multirow{2}{*}{ HS } \\
\hline & Range & $1-6$ & $2.29-4.06$ & & & \\
\hline \multirow{2}{*}{ Peripheral vascular disease } & Mean \pm SD & $2.13 \pm 1.08$ & $2.57 \pm 0.64$ & \multirow{2}{*}{-3.487} & \multirow{2}{*}{0.001} & \multirow{2}{*}{ HS } \\
\hline & Range & $1-6$ & $1.35-4.06$ & & & \\
\hline
\end{tabular}

Table 5. Comparison between the mild and moderate distress groups as regard the parameter of glycemic control (HbA1c), inflammatory marker (CRP)

\begin{tabular}{|c|c|c|c|c|c|c|}
\hline & & \multicolumn{2}{|c|}{ Total DDS score } & \multirow{2}{*}{ Test value• } & \multirow{2}{*}{ P-value } & \multirow{2}{*}{ Sig. } \\
\hline & & Mean \pm SD & Range & & & \\
\hline \multirow{2}{*}{ HbA1C } & Mild distress & $8.01 \pm 1.97$ & $5.2-15.8$ & \multirow{2}{*}{-3.720} & \multirow{2}{*}{0.000} & \multirow{2}{*}{ HS } \\
\hline & Moderate distress & $9.11 \pm 2.22$ & $6.3-14$ & & & \\
\hline \multirow{2}{*}{ CRP } & Mild distress & $3.24 \pm 1.04$ & $5.2-12.1$ & \multirow{2}{*}{2.399} & \multirow{2}{*}{0.008} & \multirow{2}{*}{ HS } \\
\hline & Moderate distress & $3.66 \pm 1.25$ & $2-6$ & & & \\
\hline
\end{tabular}

Table 6. Logistic regression analysis between moderate distress by total DDS score and diabetic complications

\begin{tabular}{|c|c|c|c|}
\hline & Odds ratio (OR) & 95\% Confidence interval (C.I) \\
\hline DKA & 2.355 & 1.096 to 5.062 \\
\hline Hypoglycemia & 2.758 & 1.225 to 6.207 \\
\hline Retinopathy & 2.836 & 1.562 to 5.150 \\
\hline Neuropathy & 2.633 & 1.382 to 5.020 \\
\hline Cardiovascular & 2.107 & 0.014 \\
\hline Cerebrovascular & 2.704 & 0.001 \\
\hline PVD & 2.025 & 1.141 to 3.891 \\
\hline
\end{tabular}

Total DDS score

\section{口Mild distress $\square$ Moderate distress}

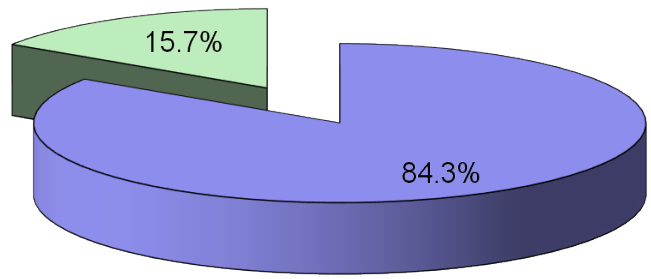

Figure 2. The percentage of mild distress group and moderate distress group as regard the total DDS score

$2.704(1.155-6.329)$ and $2.025(1.086-3.775)$ respectively.as shown in Table 6.

\section{Discussion}

The current study showed that there is a highly statistically significant positive correlation between Total DDS score and glycemic profile (FBS, 2Hpp, HbAlc) (P- value 0.000) this result came in line with [6] who found that the diabetes distress have been significantly associated with poor glycemic control and high $\mathrm{HbAlc}$, also this result was in agreement with [7] who found that Greater diabetes distress was associated with higher HbAlc.

In the present study there was a highly significant positive correlation between hs-CRP and diabetes distress this result was in agreement with [8] who found that patients with diabetes distress had high hs-CRP levels, also this result came in line with [9] who found a significant positive association between high hs-CRP levels and diabetes 
distress so suggested the possibility of a common biological pathway such as inflammation as an underlying mechanism of the association between diabetes and distress.

\section{Conclusion}

Diabetes related Distress is associated with poor glycemic control as the patients with diabetes and moderate diabetes distress have a highly significant correlation with higher HbAlc and CRP. So it is important to assess and treat people with diabetes for Diabetesrelated distress in order to identify and help them overcome barriers to optimal glycemic control.

\section{Conflict of interest}

The authors declare no conflict of interests. There was no financial funding from any institution. We declare receiving no funds or grants for this study.

\section{References}

1. American Diabetes Association (2018) Diagnosis and classification of diabetes mellitus. Diabetes Care 27: S5-S10.
2. Katon WJ, Rutter C, Simon G, Lin EH, Ludman E, et al. (2005) The association of comorbid depression with mortality in patients with type 2 diabetes. Diabetes Care 28: 2668-2672. [Crossref]

3. Snoek FJ, Bremmer MA, Hermanns N (2015) Constructs of depression and distress in diabetes: time for an appraisal. Lancet Diabetes Endocrinol 3: 450-460. [Crossref]

4. Baumeister H, Hutter N, Bengel J, Härter M (2011) Quality of life in medically il persons with comorbid mental disorders: a systematic review and meta-analysis. Psychotherapy and Psychosomatics 80: 275-286. [Crossref]

5. Fisher L, Polonsky WH, Hessler DM, Masharani U, Blumer I, et al. (2015) Understanding the sources of diabetes distress in adults with type 1 diabetes. J Diabetes Complications 29: 572-577. [Crossref]

6. Fisher L, Russell E, Strycker L (2010) The relationship between diabetes distress and clinical depression with glycemic control among patients with type 2 diabetes. Diabetes Care 33: 1034-1036. [Crossref]

7. Lee AA, Piette JD, Heisler M (2018) Diabetes distress and glycemic control: the buffering effect of autonomy support from important family members and friends. Diabetes Care 41: 1157-1163. [Crossref]

8. Yasuaki Hayashino (2018) Diabetes distress and all-cause mortality in patients with Type 2 diabetes-A prospective cohort study. Diabetes 67: 796.

9. Van Dooren FE, Nefs G, Schram MT, Verhey FR, Denollet J, et al. (2016) Psychological and personality factors in type 2 diabetes mellitus, presenting the rationale and exploratory results from the maastricht study, a population-based cohort study. BMC Psychiatry 16: 17. [Crossref]

Copyright: $@ 2019$ Hamed MS. This is an open-access article distributed under the terms of the Creative Commons Attribution License, which permits unrestricted use, distribution, and reproduction in any medium, provided the original author and source are credited. 